\title{
WHAT THE SMART CITY IN THE DANUBE REGION CAN LEARN FROM INDUSTRY 4.0
}

\author{
Alexander Prosser ${ }^{1}$
}

\begin{abstract}
The Smart City Concept throughout all its current definitions is essentially a system that uses stateof-the-art ICT to provide and process information, to adapt and learn. The Internet of Things and advances in affordable sensor technology play an additional important role. The net result of the "smartification" of a city is the creation of a living, networked system of assets, devices and infrastructure. This living system continuously collects data that enables the system to learn and evolve.
\end{abstract}

This is nothing new or path-breaking. In logistics and the manufacturing industry, this concept has been widely implemented to optimise supply chains, from predictive maintenance, to dynamic route optimisation and online business intelligence (BI). "Industry 4.0" has evolved from a buzzword to everyday reality. Moreover, these technologies do not just "electrify" existing processes - they enable new processes and beyond that even completely new business models that would not have been feasible with the pre-Industry-4.0 technology. Particularly the advent of in-memory business analytics that enables BI from the original transaction data in an on-demand/online fashion has facilitated this development. Now, the public sector is discovering these technologies for its own purposes.

This contribution attempts to show the parallelism, but also differences between smart cities and Industry 4.0, where learning effects may occur and known pitfalls may be avoided.

\section{Introduction}

\subsection{Smart City}

As with any new concept, the term Smart City has been interpreted in different ways. Some place more emphasis on inclusiveness and social openness [1], environmental and sustainability aspects may play an important role [2] and others focus on technological and energy efficiency and an "intelligent" infrastructure (cf. [4] and the definition of the Wiener Stadtwerke in [3]). ${ }^{2}$ The perhaps most widely-used definition by Frost and Sullivan encompasses "smart energy, smart building, smart mobility, smart healthcare, smart infrastructure, smart technology, smart governance, smart education, smart citizen." [5] What these definitions seem to have in common on the technological level are:

\footnotetext{
${ }^{1}$ University of Economics and Business, Vienna, Welthandelsplatz 1, A-1020 Wien

2 An extensive overview of various definitions is provided by the Smart Cities Council at https://smartcitiescouncil.com/smart-cities-information-center/definitions-and-overviews
} 
- The "smartification" of hitherto analogous infrastructure (particularly the "sensorisation of things", cf. [5]);

- $\quad$ Extensive use of ICT, particularly mobile services;

- The efficient use of resources on all levels (from staff to energy) due to intelligent adaptation of services and a focus on sustainability.

\subsection{Industry 4.0}

One has to be aware that this originally was a concept advanced in Germany [6], but has gained wide-spread usage in Europe, including the Danube Region - however, not without criticism of the terminology (for example [7]). This paper holds that, essentially, Industry 4.0 hinges on some distinct technological developments:

\subsubsection{Cheap and Connected Sensors}

The price of sensors in general and Internet-capable sensors in particularly has come down considerably. This is the commercial/technological driver of the Internet of Things (IoT). Image sensors and accelerometers on average have come down from 22 USD apiece in 1992 to 1.40 USD in 2014 (not inflation-adjusted) [8]. Goldman/Sachs estimates that the "average IoT sensor" has come down from 1.30 USD in 2004 to below 0.50 in 2018 [9]. Micro-mechanical devices (MEMS) have come down in prices from an average of approx. 3.50 USD in 2000 to below 1 USD in 2014.[10]

All these figures show a marked decrease in sensor/IoT element prices, which makes it economically viable to put a sensor behind any interesting part and to connect it to the Internet. An example would be Predictive Maintenance, where spare parts in investment goods (eg, large machinery) are monitored by IoT-connected sensors and when a threshold value of wear and tear is reached, a requirement is sent back to the logistics monitoring application. [11] This enables to minimise (ideally cut altogether) the spare part storage on site and yet to reach a near-ideal service level of the spare parts for the machinery (for a simulation study, see [12]).

\subsubsection{Cloud Services}

"The Cloud" has become yet another buzzword in enterprise computing used in many contexts, however, there is a sound technological core behind it in the context of Industry 4.0. The concept requires IoT connected modules to interact with a number of applications. Two or three decades ago this would have required enormous interface programming effort that would have stymied any commercial viability. Web services, that is SOAP-based [13] application services, enabled an object-oriented encapsulation of complete services including data storage and business logic. These encapsulated modules "export" a standardised interface in XML notation that can be "consumed" by any authorised application, whereby the latter does not need to know any details of the internal structures of the application. The web service interface, its services and expected responses are the only thing a service consumer needs to know.

This enables, for instance, a central logistics application to export a web service for reporting sensor values that (schematically) would look like the following: 
$<$ ID of reporter $>$ Machine $123.456<$ ID of reporter $>$

$<$ Value $>$ (may appear an arbitrary number of times)

$<$ SensorID $>123.456 .1</$ SensorID $>$

$<$ SensorType $>$ Accelerometer $</$ SensorType $>$

$<$ Timestamp $>$ 4.1.2018-10:23:30-CET $</$ Timestamp $>$

$<$ SensorValue $>5</$ SensorValue $>$

$<$ /Value $>$

The only things required are (i) security and authentication procedures and parameters and (ii) semantics, eg, that an accelerometer reports values to be interpreted as $\mathrm{m} / \mathrm{s}^{2}$.

Web services also help to bridge gaps between different platforms and to integrate new platforms in existing applications. It is the standardised web service architecture that completely abstracts from the internals of an application which has enabled the free flow of information among application boundaries.

\subsubsection{Real-Time Business Intelligence}

Every transactional/operational information system is essentially a huge data-generating machine collecting formatted or unformatted digital or analogous data depending on the field of application. This can be a main source of information - providing the data stream is analysed, ideally in real time. Typical methods of analysis are multi-variate statistical methods, such as clustering, loglinear/logit models, conjoint analysis or multi-variate regression (for an introduction, see [11]). These methods, however, have one main disadvantage: They require access to the raw (transactional) data, not just to aggregates.

"Classical" business intelligence has been based on data warehouses, which are essentially multidimensional aggregation hierarchies.[14] Within them, analyses are easy and flexible, typically supported by graphical data modelling and reporting tools. Data warehousing typically does not go for the raw transactional data - and for a very simple reason: The amount of data would be so big that any timely analysis would only be possible for comparatively small data sets.

It is another technical innovation that has overcome the limitations of data warehouses, the steep performance increase and price drop in main memory elements. ${ }^{3}$ This enables huge data banks being loaded into main memory. This makes a difference in access speed. The access time of a hard disk drive is measured in milliseconds $(\mathrm{ms})^{4}$, the access time of main memory banks in nanoseconds $(\mathrm{ns})^{5}$. The difference between $1 \mathrm{~ms}$ and $1 \mathrm{~ns}$ is $10^{-6}$; this means that a read operation that takes 11.5 days reading data from disk can be done in 1 second reading data from main memory. ${ }^{6}$ Concerning capacity, standardised solutions are on the market offering $64 \mathrm{~TB}$ of main memory. ${ }^{7}$ This can hold very large data banks, where the raw data can be analysed in real or near-real time without having to build inflexible aggregation hierarchies that only allow fast access to aggregate analyses like in a

\footnotetext{
${ }^{3}$ Just as a flashlight: In 2007, a 2x 1 GB DIMM DDR2 would have been at USD 130.- (or 6.6 ct per MB); in 2017 a 2 x 16GB DIMM DDR4 was at USD 185.- or $0.6 \mathrm{ct}$ per MB. Source: http://jcmit.net/memoryprice.htm

${ }^{4}$ For an example with explanations, see http://fibrevillage.com/storage/596-hard-drive-performance-in-detail-transferrates-latency-and-seek-times

${ }^{5} \mathrm{Cf}$. an overview of typical elements in https://en.wikipedia.org/wiki/DDR4_SDRAM

${ }^{6}$ Caveat: This is a schematic and illustrative example only!

${ }^{7}$ For an example, see http://e.huawei.com/en/solutions/business-needs/data-center/sap-hana/tdi
} 
data warehouse. In-memory computing offers real-time business intelligence based on unaggregated raw data.

The impact of this development on Business Intelligence cannot be overestimated.

\section{Commonalities}

The following sections will describe a number of commonalities between the two concepts.

\subsection{Efficiency Gains through a "Living" Infrastructure}

An infrastructure that is "wired" the way described Sections 1.2.1 and 1.2.2 can be constantly monitored and also returns data for optimisation which can be fed back in an optimisation loop, much the same way as MES (Manufacturing Execution Systems, for an introduction, see [15]). This optimisation loop in MES was not the original intention or focus of MES, which were originally considered the hinge between machine/infrastructure control systems (SCADA [17], cf. M1, M2, ... in Figure 1) and the planning system, typically an Enterprise Resource System (ERP). MES would assign the infrastructure, load and lock orders on the machinery and confirm execution or production order steps. It would hence enable tracking and tracing of production orders and connect the ERP system to the physical machine control.

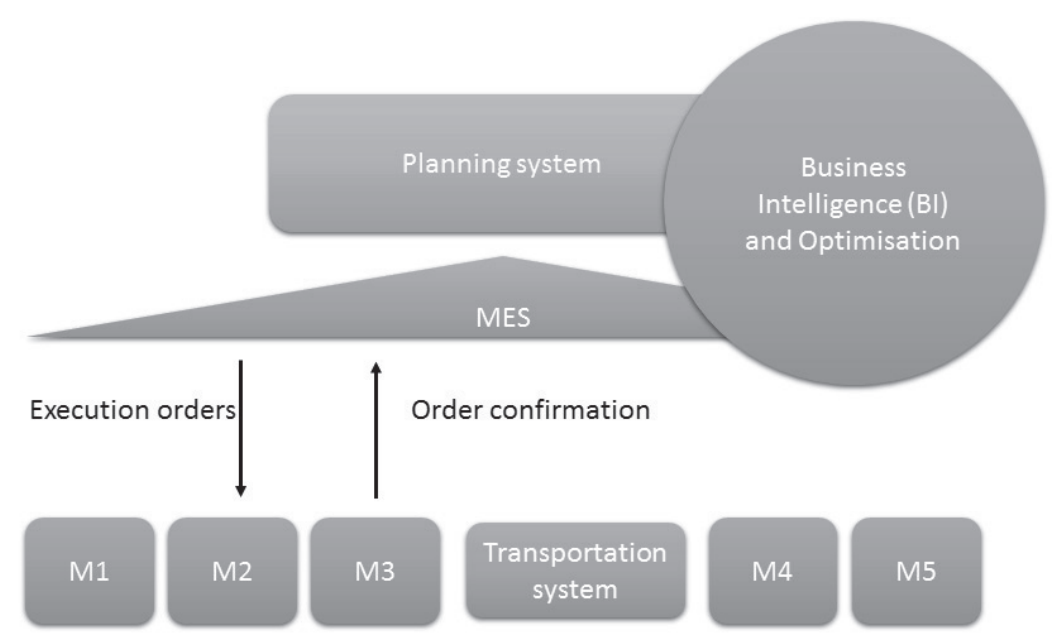

Figure 1: MES

However, the feedback of state data from the SCADA can be fed into a BI application, which then enables analyses, from a simple search for inefficiencies to the complete optimisation of the process. It can also serve as benchmarking device between comparable production facilities or to detect systematic deficiencies in production processes. Therefore, "optimisation" does not only mean optimised operational processes for, for instance, increasing machine utilisation, but also tactical optimisation by spotting and eliminating systematic deficiencies in the production process. In this context, MES have evolved into a massive data collection engine from the physical machine and transportation infrastructure that can feed its data into real-time BI. These applications are not a distant future but already in place in well-run production facilities. 
This can also be done in the Smart City; consider a simple example application intended to show the potential:

(i) Equip every available free parking spot with a sensor showing, whether it is occupied or not;

(ii) Link these sensors to a central monitoring application;

(iii) Include occupation states of parking houses, which can address the application via a cloud service;

(iv) Make the current state available to motorists via smartphone app;

(v) Provide an interface to navigation apps.

This application would correspond to what is already in place, for instance in the City of Dubrovnik [18]. It leverages a "living" infrastructure, connected via the IoT and made available via a Webbased/Smartphone app-based service.

However, this feedback loop from the infrastructure also delivers a massive data base on parking utilisation, not only in very general terms as with a simple m-parking system ${ }^{8}$, where only the total number of parking tickets is available, but down to the detailed geographical distribution of the parking usage over time. This massive data base can then be used for several optimisation purposes, such as

(i) Time distribution of the parking situation in districts and general guidance to motorists;

(ii) Real-time parking guidance via digital signposts;

(iii) Dynamic pricing of parking slots;

(iv) (Where in place,) Dynamic adaptation of city toll system.

Such applications require all elements listed in Section 1.2: IoT-enabled sensor-equipped ("living") infrastructure, cloud services and real-time BI. The applications range from isolated optimisation tolls, such as the one described to full-fledged city-wide traffic management, such as the one used in Singapore [21].

\subsection{Predictive Tools}

This learning process on the behaviour and determinants of the infrastructure can also be used to predict events in the physical infrastructure. A very popular example in the manufacturing industry is predictive maintenance. "Classical" maintenance schemes are either purely corrective (ie, the machine is repaired when it fails) or preventive (ie, regular maintenance activities according to the schedule prescribed by the manufacturer). The first incurs a potentially huge downtime of the machinery, the second is essentially wasteful as it tends to discard spares simply because the schedule prescribes their replacement and not because they really need to be replaced. [19] The

\footnotetext{
${ }^{8}$ Such as the one in place in Vienna, http://handyparken.at
} 
issue or corrective maintenance is aggravated by long-haul supply chains, where for instance the machine producer is located in the Danube Region and the machine is used in South Asia. Buffer stocks either in situ or with local/regional supply centers may alleviate the duration of the logistics chain, but still incur capital being bound in spare stocks. [20]

The "sensorisation" of the machinery and its connection to the IoT enables to query/get information (both a pull or push model are possible); whenever a threshold value is reached by a spare part, a logistics requirement is issued to a central logistics application via a cloud service, typically implemented as described in Section 1.2.2. The threshold value is configurable and will be a lot earlier in the deterioration of the part when the logistics chain is long and with a lot of variance in duration, vice versa. When the threshold value is reached and the replenishment order issued, the part itself is still functioning perfectly - it is just time to think about a replacement. To meaningfully set the threshold value data gathering and analysis from BI applications can be used plus simulation techniques. Results show that by judiciously setting the threshold for the replenishment order a service level can be reached that corresponds to almost the service level of a (hypothetical) case, where all spares are available on site (for an example with city infrastructure, see [12]).

It is immediately obvious how the Smart City may benefit from predictive schemes; whether it is infrastructure maintenance, vehicle and machine availability or the prediction of infrastructure or device utilisation, the opportunities offered by monitoring and analysing device behaviour are essential.

\subsection{New Business Models}

The ability to remotely monitor a device and its usage offers completely new business models. When integrated information systems appeared in the 1990-ies on a large scale, it was - and of course still is - common sense not just to use them to "electrify" existing processes, but to use system integration to support new processes in the organisation [22, 23]. Since observation of remote devices becomes possible via IoT-enabled sensors and cloud services, one may advance the refutable hypothesis that these technologies tend to enable a movement away from ownership to pay-per-use models: The device, the machine, the complete infrastructure as a service.

The Smart City may benefit from this development from several angles:

(i) As a customer renting devices on a pay-per-use base rather than buying ownership of the device.

The effects on capital requirements are obvious and would be a driver very similar to the private sector, particularly in times of high sovereign debt and debt ceilings, such as those of the Euro Zone. Here careful design of the contracts may be recommended, as of course the partner renting out the infrastructure will typically set a certain minimum payable usage of the device. This may be alleviated by shared devices, particularly in the case of smaller cities. Generally these pay-per-use models are slowly gaining traction in the private sector, however, also the municipal, or in general public, administration may substantially benefit from such arrangements.

(ii) As the instance letting the devices/infrastructure elements.

Car/bike sharing models clearly belong to this model, where IoT services enable tracking of the vehicles as well as per-use payment models. 
(iii) As a mediator platform.

Citizens may offer standardised or non-standardised goods in exchange for "payment" in kind; examples could be giving up one's reserved resident parking lot in exchange for a resident parking lot in another city, where municipalities provide a match-making platform. Of course, the private sector is already far ahead here with platforms, such as Uber or Airbnb - sometimes much to the chagrin of city (and tax) administrations.

\section{The Main Concern: Security}

As has been demonstrated in the past sections, there are opportunities and potential efficiency gains by introducing Industry 4.0 techniques into the Smart City. However, this will open the municipal infrastructure also to the very same security issues that also exist with the private sector - in some cases, such as the power grid, with even more damage potential. The private sector appears to have grasped the risk exposure and implementing IoT and cloud security have become major concerns of Industry 4.0 users. This concerns (i) the integrity of the distributed systems and (ii) the data communications between the distributed, IoT-driven data banks and central (cloud) applications collecting and analysing the data (for an introduction, see [24, 25]). Apart from that, general risks of any ICT application, such as Denial of Service Attacks, apply.

The fundamental issue with IoT-enabled and intelligent infrastructure/devices is that these devices are still produced by traditional manufacturers that have little experience with ICT. Particularly the following issues can be identified:

(i) Hardening of external interfaces, such as SOAP services [13];

(ii) Judicious use of standard tools, such as TLS (SSL) [26];

(iii) A regular process for producing and distributing upgrades and patches to the IoT devices;

(iv) Upgrades and patches to the operating system and system components used;

(v) Usage of a virus scanner;

(vi) At times, the most basic elements of systems operations are not heeded, such as enforcement of password resets.

The risk is clearly identified and concerns two areas:

(i) The smart infrastructure may be used as an entrance to backend systems, this particularly applies to the power grid;

(ii) The smart infrastructure may be used for all sorts of criminal and illicit activities. ${ }^{9}$

Many of these issues can be resolved by simply adopting standard ICT practices also to the world of smart devices, others may only be resolved by requesting a security certification. If devices are

9 As an example: For some scenarios of using a smart refrigerator for terrorist activities, see https://www.wu.ac.at/en/evoting/news-details/detail/eudem-2016/ 
critical, such as smart meters potentially opening the path to the power grid, it would seem indicated to request Common Criteria certification [27].

However, such certification would be costly and time-consuming depending on the assurance level required (ie, the stringency of the tests) and will probably only be realised if mandatorily requested by standards set on a European level. Examples show however, that such assurance is sorely needed $[28,29]$.

\section{The Danube Region}

These considerations of course apply universally. However, things are aggravated in the Danube Region due to the fragmented character of the Region. Since the markets are too small to justify adaptation to national regulations, only supranational regulations may have the necessary effect. The security certifications discussed in Section 3, for example, can only realistically be implemented on an EU level, national regulations would fail due to small market size.

Bringing this topic on the EU agenda and not attempting purely national solutions is therefore in the well-understood interest particularly of the countries in the Danube Region.

\section{Summary}

The past paragraphs outlined the huge potential in the Smart City concept, its parallelism to Industry 4.0 and the technological developments underlying both. Industry 4.0 involves huge productivity gains in the manufacturing industry, there is no doubt that similar benefits may be reaped by public administration, particularly on the municipal level. Also, the technology enables completely new business models and a completely new view on the physical products of a manufacturer. The main development here is the movement from the device as a product to the device as a service - a development that has already been seen in the software industry in the past decade [30].

However, there are also differences:

(i) The driver in the manufacturing industry is mainly efficiency gains - which may of course also involve a more sustainably and more environmentally friendly way of production, customer satisfaction is a main driver, yet only one of many factors; the Smart City driver on the other hand is primarily citizen/business satisfaction and the improvement of the competitiveness of the city as a location;

(ii) Manufacturing applications of IoT and Cloud Services seldom involve personal data; if they do, a work council agreement may be drafted to enable processing of such data depending on the legal situation of the country in question; in the city context many applications will involve personal data from citizens/consumers, whereby in many cases, the data collection and processing will be "public", ie based on sensors in the public sphere, where people cannot escape the processing; this involves additional thought, particularly under the conditions of the General Data Protection Regulation, which will soon be in effect. 


\section{References}

[1] BEINROTT, V., Bürgerorientierte Smart City - Potentiale und Herausforderungen, Zeppelin Universität Friedrichshafen, Friedrichshafen, 2015, download from https://www.zu.de/institute/togi/assets/pdf/TOGI-150302-TOGI-Band-12-BeinrottBuergerorientierte-SmartCity-V1.pdf.

[2] Neirotti, P. De marco, A., CAgliano, A.C., MAngano, G., Scorrano, F., Current trends in Smart City initiatives: Some stylised facts, Cities, 38(2014), pp. 25-36.

[3] ROHDE F., LOEW, T., Smart City - Begriff, Charakteristika und Beispiele , Wiener Stadtwerke Holding AG, City of Vienna, 2011, download from http://www.nachhaltigkeit .wienerstadtwerke.at/fileadmin/user_upload/Downloadbereich/WSTW2011_Smart_CityBegriff_Charakteristika_und_Beispiēe.pdf

[4] KOMNINOS, N., What makes cities intelligent?, in Deakin, M., Smart Cities: Governing, Modelling and Analysing the Transition, Taylor and Francis, 2013.

[5] FROST \& SULLIVAN, Global Smart Cities market to reach US\$1.56 trillion by 2020, 2014, at https://ww2.frost.com/news/press-releases/frost-sullivan-global-smart-cities-market-reachus156-trillion-2020

[6] SPATH, D. (ED.), GANSCHAR, O., GERLACH, S., HÄMMERLE, M., KRAUSE, T., SCHLUND, S.: Produktionsarbeit der Zukunft - Industrie 4.0, Fraunhofer IAO, 2013, download from https:/www.iao.fraunhofer.de/images/iao-news/produktionsarbeit-der-zukunft .pdf

[7] GARBEE, E., This is not the fourth industrial revolution, at http://www.slate.com/ $\operatorname{articles} /$ technology/future tense/2016/01/the_world_economic_forum_is_wrong_this_isn_t_t he_fourth_industrial_revolution.html

[8] HOLDOWSKY, J., MAHTO, M., RAYNOR, M.E., COTTELEER, M., Inside the Internet of Things (IOT), Deloitte, 2015, download from https://www2.deloitte.com/insights/us/en/focus/ internet-of-things/iot-primer-iot-technologies-applications.html

[9] GOLDMAN/SACHS, The average cost of IoT sensors is falling, download from https://www. theatlas.com/charts/BJsmCFAl, 2016.

[10] PIZZAGALLI, A., Mems and sensors packaging technology and trends, Presentation at Semicon West, 2016, download at https://www.slideshare.net/Yole_Developpement $/ \mathrm{mems-}$ sensors-packaging-waferlevelpackaging-technology-and-market-trends-presentation-heldbyamandine-pizzagalli-on-semicon-west-2016-in-singapore-presentation-by-yoledveloppement

[11] BACKHAUS, K., ERICHSON, B., PLINKE, W., WEIBER, R., Multivariate Analysemethoden, 6th Ed., Springer, 1990. 
[12] PROSSER, A., WIJAYALATH, L.D., Simulation-based Analysis of Device Availability under three Maintenance Strategies, Transylvanian Review of Administrative Sciences, to be published.

[13] W3C, SOAP Version 1.2 Part 1: Messaging Framework (Second Edition), W3C, 2007, download from https://www.w3.org/TR/soap12/

[14] PROSSER, A., OSSIMITZ, M.-L., Data Warehouse Management Using SAP BW, UTB, 2001.

[15] KLETTI, J. (ed.), MES-Manufacturing Execution System, $2^{\text {nd }}$ Ed., Springer, 2015.

[16] ALMADA-LOBO, F., The Industry 4.0 revolution and the future of Manufacturing Execution Systems (MES), in Journal of Innovation Management 3/4 (2015), pp. 16-21.

[17] BOYER, S.A., SCADA: Supervisory Control and Data Acquisition, $4^{\text {th }}$ Ed., International Society of Automation, 2010.

[18] ŠARIĆ, A., MIHALJEVIĆ, B., Smart Parking System in the City of Dubrovnik, download from http://www.rithink.hr/brochure/pdf/vol6_2017/1509301359_4_Andrej_ari Branko_Mihaljevi_SMART_PARKING_SYYSTEM_IN_THE_CITY_OF_DUBROVNIK.p df

[19] CHOPRA, S., MEINDL, P., Supply Chain Management - Strategie, Planung und Umsetzung, Pearson, 2014.

[20] DUFFUAA, S. O., RAOUF, A., Planning and Control of Maintenance Systems: Modelling and Analysis. $2^{\text {nd }}$ ed., Springer, 1999.

[21] LAND TRAFFIC AUTHORITY SINGAPORE, Intelligent Transport Systems, at https://www.lta.gov.sg/content/ltaweb/en/roads-and-motoring/managing-traffic-andcongestion/intelligent-transport-systems.html

[22] SCHEER, A.W., ARIS - Vom Geschäftsprozess zum Anwendungssystem, $4^{\text {th }}$ ed., Springer, 2002.

[23] PROSSER, A., BAGNATO, D., MÜLLER-TÖRÖK, R., Integration Management with SAP ECC, $3^{\text {rd }}$ Ed., Facultas, 2017.

[24] WASLO, R., LEWIS, T., HAJJ, R., CARTON, R., Industry 4.0 and Cybersecurity Managing risk in an age of connected production, Deloitte, 2017, download from https://www2.deloitte.com/insights/us/en/focus/industry-4-0/cybersecurity-managing-risk-inage-of-connected-production.html

[25] PROSSER, A., BAGNATO, D., MÜLLER-TÖRÖK, R., The Cryptographic Requirements for Predictive Remote Maintenance Schemes, Transylvanian Review of Administrative Sciences, to be published. 
[26] W3C, The Transport Layer Security (TLS) Protocol Version 1.2, W3C, 2008, download from https://tools.ietf.org/html/rfc5246

[27] CCRA, Common Criteria for Information Technology Security Evaluation, Part 1: Introduction and general model, Version 3.1, Revision 5, 2017, download from https://www.commoncriteriaportal.org/cc/

[28] ROSS, M., IoT-Sicherheitskonferenz: Unsichere Smart-Meter, Mirai und seine Klone und die Genfer Konvention, download from https://www.heise.de/ix/meldung/IoT-

Sicherheitskonferenz-Unsichere-Smart-Meter-Mirai-und-seine-Klone-und-die-GenferKonvention-3872793.html

[29] THE GUARDIAN, Smart electricity meters can be dangerously insecure, warns expert, download from https://www.theguardian.com/technology/2016/dec/29/smart-electricitymeters-dangerously-insecure-hackers

[30] TURNER, M., BUDGEN D., BRERETON, P., Turning software into a service, Computer 36(10), 2003, pp. 38-44. 